\title{
0 uso da programação inteira 0-1 para 0 balanceamento de linhas de montagem: modelagem, estudos de caso e avaliação
}

Flávio César Faria Fernandes UFSCar

Moacir Godinho Filho UFSCar

Ricardo Augusto Cutigi UFSCar

Aline Malerbo Guiguet UFSCar

\section{RESUMO}

O presente trabalho trata do problema do balanceamento de linha de montagem. Dentro deste contexto o objetivo é mostrar que nos dias atuais, em muitos casos práticos, é possível, mesmo em problemas relativamente grandes, a aplicação de procedimentos de solução exatos (no caso, programação inteira). Isto é feito por meio de dois estudos de caso em grandes empresas do Estado de São Paulo, as quais apresentam problemas de balanceamento de linha relativamente grandes (linhas com 80 e 104 tarefas). Em um destes problemas foi incluída no modelo uma restrição, inédita na literatura, para melhor adequá-lo à situação real. Esta restrição se refere a situações nas quais a linha de montagem possui tanto operações manuais quanto automáticas. Os resultados conseguidos foram muito bons, conseguindo-se redução de mais de 10\% no número de estações de trabalho necessárias em cada caso.

\section{Assembly line balancing by means of integer programming 0-7: modeling, case studies and evaluation}

\begin{abstract}
This paper deals with the assembly line balancing problem. Within this context, the objective is to show that nowadays, in many practical cases, it is possible, even in large problems, the application of exact solution procedures (in this case, integer programming). This is accomplished by means of two case studies in big companies of São Paulo State, which presents large assembly line balancing problems (lines with 80 and 104 tasks). In one of these cases one constraint unpublished on literature was included in the model to adapt the model to the real situation. This constraint accounts to those cases in which the assembly line has both manual and automatic tasks. The results were very good, and a reduction of more than 10\% was attained in the number of workstations required on the assembly line.
\end{abstract}

KEY WORDS

Assembly line balancing problem, exact solution procedures, integer programming, case studies. 


\section{INTRODUÇÃO E DEFINIÇÃO DO PROBLEMA}

Uma linha de montagem pode ser definida como um conjunto finito de elementos de trabalho ou tarefas, cada uma tendo um tempo de processamento de operação e um conjunto de relações de precedência que especificam a ordem permitida das tarefas (GHOSH; GAGNON, 1989). O problema fundamental de uma linha de montagem é conhecido na literatura pela sigla ALBP (assembly line balancing problem), que consiste em atribuir as tarefas a um número ordenado de estações de trabalho, de tal forma que as restrições de precedência entre as tarefas sejam respeitadas e alguma medida de eficiência (por exemplo, minimização do número de estações de trabalho, dentre outras) seja otimizada. Segundo Fernandes \& Morábito (1993), uma linha está idealmente balanceada quando o tempo de ciclo for igual a todos os tempos que o produto gasta em cada uma das estações.

De acordo com Ghosh \& Gagnon (1989) a primeira publicação a respeito do problema de balanceamento de linha foi de Helgeson et al. (1954), cabendo a Salveson (1955) a

primeira publicação na forma matemática e uma sugestão de solução utilizando programação linear. A partir deste trabalho o assunto de balanceamento de linha vem recebendo grande interesse da comunidade científica voltada à Gestão da Produção (EREL; SARIN, 1998).

Na literatura, merecem destaque pelos menos duas classificações a respeito dos problemas de balanceamento de linha. A primeira delas divide os problemas de balanceamento de linha de montagem em quatro categorias:

i) problemas para um único modelo com tempos das tarefas determinísticos (single-model deterministic, sigla SMD);

ii) problemas para um único modelo com tempos de tarefas estocásticos (single-model stochastic, sigla SMS);

iii)problemas para múltiplos modelos com tempos das tarefas determinísticos (multi-model deterministic, sigla MMD);

iv) problemas para múltiplos modelos e tempos das tarefas estocásticos (multi-model stochastic, sigla MMS).

Outra classificação bastante importante divide os problemas de balanceamento de linha em duas categorias:

i) problema de balanceamento de linha de montagem simples (simple assembly line balancing problem, sigla SALBP): onde o termo simple indica que nenhuma restrição é relaxada; na seção 2.1 apresentamos as hipóteses assumidas pelos SALBP. ii) problema de balanceamento de linha de montagem generalizado (generalized assembly line balancing problem, sigla GALBP): onde se encaixam os problemas de balanceamento de linha que objetivam resolver problemas com algumas características adicionais (inclusive algumas restrições do SALBP podem ser relaxadas) tais como: a) possibilidade de seleção de equipamentos ou de processos; b) agregação de estações de trabalho ou estações de trabalho com máquinas em paralelo; c) linha de montagem com layout em formato U; d) tempos de tarefas estocásticos; e) recursos limitados; (f) outras características.

O foco deste trabalho é somente o SMD, sendo que um dos dois problemas apresentados é um SALBP e o outro é um GALBP.

\section{presente trabalho objetiva mostrar que nos dias mesmo em problemas relativamente grandes, a aplicação de modelos exatos de programação inteira.}

De acordo com Erel \& Sarin (1998) existem basicamente duas abordagens de solução para o SALBP: a) métodos exatos ou ótimos; b) algoritmos heurísticos (para uma comparação entre métodos heurísticos ver Talbot et al., 1986). Para uma revisão referente aos métodos de solução do SALBP, ver Scholl \& Becker (2006). O SALBP é reconhecidamente um problema de otimização combinatória do tipo NP-hard (EREL; SARIN, 1998; SCHOLL, 1999; SCHOLL; BECKER, 2006). Portanto, segundo a literatura atual, a utilização de métodos exatos para a solução de grandes problemas na prática se torna bastante difícil computacionalmente.

Dentro deste contexto, o presente trabalho objetiva mostrar que nos dias atuais, em muitos casos práticos, é possível, mesmo em problemas relativamente grandes, a aplicação de modelos exatos de programação inteira que levem a um resultado ótimo. Isto é feito por meio de dois estudos de caso em grandes empresas do Estado de São Paulo, as quais apresentam um GALBP ou SALPB relativamente grande (linhas com 80 e 104 tarefas). O modelo exato aplicado nos dois casos é o modelo proposto originalmente por Thangavelu \& Shetty (1971) e aperfeiçoado por Fernandes \& Morábito (1993). A este modelo aperfeiçoado, foi incluída, em um dos casos, uma restrição inédita na literatura para melhor adequar o modelo à situação real. Esta restrição se refere a situações nas quais a linha de montagem possui tanto operações manuais quanto automáticas. 
As técnicas formais de balanceamento de linha apresentam freqüentemente dificuldades em serem utilizadas na prática. Este trabalho contribui neste sentido, uma vez que adapta um modelo exato a situações reais e mostra que resultados excelentes podem ser alcançados em situações nas quais acreditava-se que métodos ótimos não poderiam ser aplicados.

A estrutura do trabalho é a que segue: na seção 2 são descritas as hipóteses necessárias ao SALBP, e mencionados os principais modelos de solução exata para estes problemas, dando destaque ao modelo utilizado nos estudos de caso. Também nesta seção é descrita a dificuldade computacional mencionada na literatura na solução exata de problemas SALBP. Na seção 3 é mostrada a aplicação do método proposto em dois estudos de caso, o primeiro deles em uma grande fábrica de motores e o segundo em uma grande fábrica da linha branca. Na seção 4 são mostrados os resultados e a avaliação dos estudos de caso. Na seção 5 são tecidas algumas conclusões.

\section{MODELOS DE SOLUÇÃO EXATA PARA O SALBP}

\subsection{As hipótese assumidas nos SALBP}

Autores como Becker \& Scholl (2006); Scholl (1999); Baybars (1986), dentre outros, descrevem as hipóteses que são assumidas nos SALBP:

H1) todos os parâmetros de entrada são conhecidos com certeza;

H2) uma tarefa não pode ser dividida entre duas ou mais estações;

H3) a alocação de tarefas a estações deve respeitar as restrições tecnológicas de precedência;

H4) todas as tarefas devem ser alocadas e portanto processadas;

H5) qualquer estação possui capacidade tecnológica para processar qualquer tarefa (o efeito disso é que os custos fixos e variáveis de processamento em todas as estações de trabalho são os mesmos e portanto não precisam ser levados em consideração no modelo) ;

H6) o tempo de processamento de qualquer tarefa é independente de qual estação executará e independente da seqüência de processamento da estação;

H7) qualquer tarefa pode ser processada em qualquer estação;

H8) a linha toda é considerada serial e não existem estações em paralelo, sublinhas alimentadoras ou estações de duplo lado;

H9) a linha só montará um único modelo de produto; H10) Os principais critérios de otimização são: (i) mi- nimizar o tempo ocioso total para um dado tempo de ciclo fixo (o que é equivalente a minimizar o número de estações de trabalho); (ii) minimizar o tempo de ciclo para um dado número fixo de estações de trabalho (o que é equivalente a maximizar a taxa de produção).

Caso o SALBP tenha como critério de otimização o critério (i) então ele é conhecido como SALBP-1 ou então problema do tipo I. Caso o SALBP tenha como critério de otimização o critério (ii) então o SALBP é conhecido como SALBP-2 ou problema do tipo II. Caso o objetivo seja a otimização de ambos os critérios, o problema é dito SALBP-E (ver PLANS; COROMINAS, 1999). Nestes três casos o problema de balanceamento de linha é dito orientado ao tempo. Em casos onde o problema busca a minimização de custos o problema é chamado de problema de balanceamento de linha orientado ao custo (cost-oriented assembly line balancing problem). Exemplos de trabalhos desta natureza são: Amen (2001, 2000). Amen (2006) apresenta uma revisão sobre as diferentes formulações e métodos de solução deste tipo específico de problema.

Neste trabalho, tratamos de um objetivo relacionado ao tempo, a minimização do tempo ocioso total para um dado tempo de ciclo fixo (o que é equivalente a minimizar o número de estações de trabalho); portanto, tratamos do SALBP-1.

A seguir apresentamos algumas das principais modelagens de solução exata para o SALBP-1 mostradas na literatura.

\subsection{Uma visão geral da literatura sobre modelos de solução exata para o SALBP-1}

Salveson (1955) foi o primeiro autor a formular matematicamente o SALBP-1. Porém sua formulação não foi em programação inteira, resultando em algumas soluções inviáveis. Bowman (1960) foi o primeiro autor a tratar o SALPB-1 como um problema de programação inteira. Este modelo sofreu algumas alterações sugeridas por White (1961). Baybars (1986) denomina este modelo de modelo BW. Basicamente o modelo BW busca a minimização de uma função objetivo que representa o custo total de se utilizar mais estações de trabalho do que o mínimo necessário. As restrições (além da restrição de programação do tipo 0-1) são de três tipos: i) restrições que certificam que todas as tarefas são executadas (chamadas restrições de ocorrência); ii) restrições que garantem que o conteúdo de trabalho em qualquer estação de trabalho não excede o valor do tempo de ciclo; iii) restrições de precedência entre as tarefas. Estes três tipos de restrições são as restrições básicas encontradas nos problemas SALBP-1 (EREL; SARIN, 1998). 
O modelo BW sofreu uma série de alterações visando a redução da complexidade do problema. Thangavelu \& Shetty (1971) modificaram a função objetivo, as restrições de ocorrência e as restrições de precedência. Patterson \& Albracht (1975) também realizaram modificações na função objetivo e nas restrições de ocorrência e procedência. De acordo com Baybars (1986) estas modificações reduziram consideravelmente o tamanho das formulações do SALBP-1.

Fernandes \& Morábito (1993) apresentaram um aperfeiçoamento do modelo de Thangavelu \& Shetty (1971). É exatamente este modelo (com o acréscimo de um conjunto de restrições para o primeiro estudo de caso) que será utilizado neste trabalho. A seguir mostramos este modelo.

Definem-se os índices $\mathrm{i}=1,2, \ldots, \mathrm{n}$ representando cada tarefa e $\mathrm{j}=1,2, \ldots, \mathrm{k}$ representando cada estação de trabalho. Sejam:

$\mathrm{x}_{\mathrm{ij}}=1$, se a tarefa i for atribuída a estação de trabalho j;

0 , caso contrário.

Seja MATRIZ, uma matriz representando as restrições de precedência entre as tarefas. A tarefa u deve preceder imediatamente a tarefa $\mathrm{v}$ se e somente se MATRIZ $(\mathrm{u}, \mathrm{v})=1$.

O objetivo é minimizar o número de estações necessárias. A função objetivo originalmente proposta por Thangavelu \& Shetty (1971) foi modificada por Fernandes \& Morábito (1993) para:

$$
\operatorname{Min} \sum_{j=1}^{k} \sum_{i=1}^{n} d_{j} x_{i j}
$$

onde:

$d j=>$ são coeficientes arbitrados de forma crescente (isto é;

$\left.d_{1}<<d_{2}<<\ldots<<d_{k}\right)$,

representam penalidades para o caso de se utilizar um número de estações de trabalho maior do que o mínimo necessário.

Lembrando que:
Número mínimo de estações trabalho $=\frac{\sum_{i=1}^{N} p_{i}}{C}$

onde:

$C=$ tempo de ciclo (não pode ser menor que o tempo de montagem do posto mais lento)

$$
\sum_{i=1}^{N} p_{i}=\text { tempo total de trbalho requerido por um item }
$$

Quanto às restrições, temos quatro tipos de restrições:
1 - Restrições da programação inteira do tipo 0-1:

$$
x_{i j}=0,1 \text { para todo } i \text { e para todo } j
$$

2 - A soma dos tempos de processamento das tarefas nas estações deve ser menor ou igual do que o tempo de ciclo C. Isto pode ser escrito como:

$$
\sum_{i=1}^{n} p_{i} x_{i j} \leq C \text { para todo } j
$$

3 - Toda tarefa deve ser realizada em alguma estação. Isto pode ser escrito como:

$$
\sum_{j=1}^{k} x_{i j}=1 \text { para todo } i
$$

4 - Se a MATRIZ (u,v) = 1, então a tarefa u deve preceder a tarefa v e vice-versa. Fernandes \& Morábito (1993) modelam esta restrição simplificando Thangavelu \& Shetty (1971):

$$
\sum_{j=1}^{k} j x_{u j} \leq \sum_{j=1}^{k} j x_{v j} \begin{aligned}
& \text { para }(u, v) \text { tal que MATRIZ } \\
& (u, v)=1
\end{aligned}
$$

Observe que o lado esquerdo das inequações acima fornece o número da estação de trabalho em que a tarefa u foi alocada e o lado direito fornece este número para a tarefa v. Assim sendo, fica garantido que u precede v.

Fernandes \& Morábito (1993) acreditam que estas restrições possuem duas vantagens em relação às restrições propostas por Thangavelu \& Shetty (1971): (i) o número de termos é menor em cada restrição; (ii) possuem um "significado físico" mais evidente.

Qualquer um dos modelos de programação inteira citados fornece uma solução ótima para o SALBP-1. De acordo com a literatura, o grande problema destes modelos é a complexidade computacional, uma vez que o SALBP é reconhecidamente um problema de otimização combinatória do tipo NP-hard, sendo que o tempo computacional cresce exponencialmente com o número de tarefas. Portanto, de acordo com a literatura, a utilização na prática destes métodos para a solução de problemas com um número médio ou grande de tarefas (acima de 30 tarefas, por exemplo) se torna inviável. Outros fatores que aumentam a complexidade computacional dos SALBP são: i) número de relações de precedência (chamado força da seqüência por Mastor, $1970 \mathrm{e}$ taxa de flexibilidade por Dar-El, 1973); ii) número de tarefas por estação na solução ótima. Na próxima seção mostramos dois estudos de caso reais, com 80 e 104 tarefas, respecti- 
vamente, que foram (por questões tecnológicas) divididos em sublinhas menores, e assim puderam ser resolvidos de forma exata.

\section{A APLICAÇÃO DO MODELO PROPOSTO EM DOIS ESTUDOS DE CASO}

\subsection{A aplicação em uma fábrica de motores (estudo de caso 1)}

\subsubsection{A linha de montagem estudada}

A linha de montagem estudada (EA-111) é responsável pela montagem de uma família de motores. Os motores que compõem esta família são: Motor 1.0L 8 Válvulas RSH Longitudinal (Gasolina); Motor 1.0L 8 Válvulas Tasse (Álcool e Gasolina ;) Motor 1.0L 8 Válvulas RSH Transversal (Gasolina, Bicombustível ); Motor 1.0L 16 Válvulas RSH (Gasolina); Motor 1.0L 16 Válvulas Turbo (Gasolina); Motor 1.4L 8 Válvulas RSH Exportação (Gasolina); Motor 1.6L 8 Válvulas RSH (Gasolina, Bicombustível).

A linha de montagem trabalha com um sistema de transporte por pallets onde os mesmos percorrem trilhos e são movimentados por correntes. Cada pallet possui dois pinos de fixação (index) que garantem o alinhamento do motor para as operações automáticas. Os pallets também contam com um sistema de movimentação giratório que possibilita ao montador dar um giro de $360^{\circ}$ no motor.

A linha recebe um motor parcialmente montado, ou subconjunto (Chamado Rumpfmotor) proveniente de uma linha de montagem anterior. Este motor parcial contempla todos os componentes internos do motor como virabrequim, bloco, bielas, pistões, cabeçote e cárter. Com a recepção do motor parcial, a linha terá a função de montagem dos periféricos do motor, tornando-o um motor completo (Chamado de ZP4).

A linha é monitorada por um painel central e todos os postos de trabalho estão conectados ao painel central. Cada pallet transportador possui um dispositivo de armazenamento de dados eletrônicos (chamado Moby I). Este Moby I funciona como um disquete que é carregado pelo pallet e em cada estação existe um leitor que verifica se o motor que está naquele pallet possui tarefas a serem realizadas naquela estação. Em caso positivo, o motor permanece parado na estação e automaticamente transmite as informações específicas do motor em operação para as torqueadeiras e para o Painel de Controle do Posto.

A informação contida em cada Moby I está relacionada ao motor que o pallet transporta. A informação é inserida no armazenador

Apesar desta diversidade de modelos eles podem ser entendidos como um modelo único, pois os diversos tipos têm características técnicas em comum, como por exemplo dimensões de componentes que são padrão da marca. Algumas pequenas particularidades de cada modelo de motor são absorvidas por postos de trabalho flexíveis (chamados de postos manuais) onde a adaptação do posto de trabalho para diferentes componentes da montagem se torna possível.

A linha de montagem conta atualmente com 40 estações de trabalho, sendo que 31 são manuais e 9 possuem operações automáticas. A linha é dividida em três células de trabalho, sendo que esta divisão tem o objetivo de facilitar a administração da mão-de-obra e já leva em conta uma imposição tecnológica (por exemplo, peças da parte inferior do motor devem ser montadas na mesma sublinha - célula, conforme a nomenclatura adotada na empresa). Assim, o balanceamento utilizando o modelo proposto será feito separadamente para cada uma das três "células", as quais chamaremos de sublinhas 1,2 e 3 . eletrônico no início da linha de montagem e no último posto de trabalho da linha (posto de retirada do motor) a informação é apagada, deixando o Moby I pronto para uma nova gravação.

Os postos de trabalho, após receberem as informações do Moby I , liberam apenas as tarefas que são necessárias para a montagem do motor em específico, ou seja, se um motor necessita receber três torques em uma determinada estação, a torqueadeira e o painel de controle recebem exatamente esta informação e garantem que o motor somente poderá ser liberado do posto após ter recebido os três torques de acordo com as especificações de engenharia. Este processo funciona como um monitoramento da realização das tarefas pelo montador, impossibilitando a liberação de um motor fora de especificação.

A taxa de produção é de aproximadamente 75,87 motores por hora. O tempo de ciclo (C) é dado por:

$$
C=\frac{1}{\text { taxa de produção }}=\frac{1}{75,87}=47,45 \mathrm{~s}
$$


Este tempo de ciclo refere-se ao teste a frio do motor (Kalt Test), que é uma estação de trabalho automática. Esta estação origina três fatores responsáveis pelo alto índice de desbalanceamento da linha:

a) a estação é localizada no centro da linha de montagem;

b) uma grande quantidade de componentes deve ser mon-

tada antes dessa estação pois os mesmos fazem parte dos testes;

c) alto tempo de processamento do teste a frio.

Na tabela 1 apresentamos as tarefas (com seus respectivos tempos) e relações de precedência para cada uma das três sublinhas estudadas. Na sublinha 1 tem-se 19 tarefas manuais (1 a 19) e 4 automáticas (A, D, C e D). Na sublinha 2 tem-se 22 tarefas manuais $(20$ a 41$)$ e 3 automáticas (E, $F$ e G). Na sublinha 3 tem-se 30 tarefas manuais ( 42 a 71 ) e 2 automáticas (H e I). A situação atual do balanceamento de linha na empresa é a seguinte: o número de estações de trabalho necessário em cada uma das três sublinhas é igual a 13,12 e 15 , respectivamente.

\subsubsection{A modelagem específica para o estudo de 1}

O modelo utilizado no estudo de caso é o modelo proposto por Fernandes \& Morábito (1993) e mostrado na seção 2.2. Porém, no estudo de caso 1 ocorre uma restrição adicional, e portanto o caso é do tipo GALBP. Esta restrição se deve ao fato de existirem na linha de montagem em questão, além das tarefas manuais, também tarefas automáticas. Cada uma das tarefas automáticas deve ser atribuída a somente uma estação de trabalho, sendo que esta estação não deverá realizar mais nenhuma tarefa, mesmo que o tempo de execução da tarefa seja bem menor que o tempo de ciclo. O modelo proposto por Fernandes \& Morábito (1993) não considera esta questão. Portanto, para o presente caso deve-se adicionar ao modelo matemático de Fernandes \& Morábito (1993) um conjunto de restrições que considerem esta situação.

A modelagem desta restrição segue:

Suponha que a tarefa arbitrária 5 seja uma tarefa automática. Caso ela seja atribuída à estação j', temos que mais nenhuma tarefa pode ser atribuída à estação j'. Isto pode ser expresso como:

$$
\text { Se } x_{5 j^{\prime}}=1 \text { então } \sum_{i=1}^{n} x_{i j^{\prime}}=1
$$

Caso a tarefa 5 não seja atribuída à estação j', temos que a estação $j$ ' pode receber qualquer número de tarefas (devemos lembrar que este número não poderá ser superior a um número $\mathrm{M})$. Isto pode ser expresso como:

$$
\text { Se } x_{5 j^{\prime}}=0 \text { então } \sum_{i=1}^{n} x_{i j^{\prime}}>0
$$

As duas condições acima podem ser escritas por meio da seguinte restrição:

$$
(M-1) x_{5 j}+\sum_{i=1}^{n} x_{i j} \leq M \quad \text { para todo } j
$$

onde:

$$
M=\frac{c}{\min \left(p_{i}\right)}
$$

A situação modelada é inédita em termos de literatura e é uma realidade não somente para a linha de montagem estudada, mas também para uma grande parte das linhas de montagem existentes atualmente. Mais especificamente: a) a restrição adicional proposta neste estudo de caso por si só representa uma contribuição, uma vez que pode ser utilizada na prática em problemas de balanceamento de linhas que apresentam tarefas manuais e automáticas; b) não encontramos modelagem com estas características em uma análise da literatura sobre o GALBP (um survey bastante recente sobre o GALBP, encontrado em Becker \& Scholl (2006) não mostra esta situação específica).

\subsection{A aplicação em uma indústria do segmento de linha branca (estudo de caso 2)}

\subsubsection{A linha de montagem estudada}

A linha de montagem possui layout do tipo linear ou por produto. É dividida em 6 sublinhas, operadas por equipe de trabalho, onde cada tarefa é atribuída previamente ao operador. Em cada sublinha há rotações de trabalho entre os operadores. Atualmente possui uma taxa de produção de 150 produtos por hora.

Os operadores se dispõem ao longo da linha de montagem, composta por uma esteira transportadora, e seus postos de trabalho (onde realizam suas operações) obedecem à seqüência de processamento do produto.

O tempo de ciclo (C) é dado por:

$$
C=\frac{1}{\text { taxa de produção }}=\frac{1}{150}=24 \mathrm{~s}
$$

A linha é dividida em 6 sublinhas num total de 41 estações de trabalho. Para o nosso estudo, as sublinhas 4 e 5 não serão consideradas devido à impossibilidade de realocação das operações realizadas. O número de estações 
de trabalho em cada sublinha estudada é o seguinte: na sublinha 1 existem 8 estações para trabalhar 20 tarefas ( 1 a 20), na sublinha 2 são 9 estações para trabalhar 21 tarefas
(21 a 41), na sublinha 3 existem 12 estações para trabalhar 32 tarefas (42 a 73) e finalmente na sublinha 6 existem 12 estações para trabalhar 31 tarefas (74 a 104). Na tabela 2

Tabela 1: Tempo médio das tarefas relações de precedência das sublinhas 1, 2 e 3.

\begin{tabular}{|c|c|c|c|c|c|c|c|c|}
\hline \multicolumn{3}{|c|}{ SUBLINHA 1} & \multicolumn{3}{|c|}{ SUBLINHA 2} & \multicolumn{3}{|c|}{ SUBLINHA 3} \\
\hline $\begin{array}{l}\text { Código } \\
\text { Tarefa }\end{array}$ & $\begin{array}{l}\text { Tempo } \\
\text { Tarefa } \\
\text { (seg.) }\end{array}$ & $\begin{array}{c}\text { Precede } \\
\text { diretamente } \\
\text { atividades }\end{array}$ & $\begin{array}{l}\text { Código } \\
\text { Tarefa }\end{array}$ & $\begin{array}{c}\text { Tempo Tarefa } \\
\text { (seg.) }\end{array}$ & $\begin{array}{c}\text { Precede } \\
\text { diretamente } \\
\text { atividades }\end{array}$ & $\begin{array}{l}\text { Código } \\
\text { Tarefa }\end{array}$ & $\begin{array}{l}\text { Tempo } \\
\text { Tarefa } \\
\text { (seg.) }\end{array}$ & $\begin{array}{c}\text { Precede } \\
\text { diretamente } \\
\text { atividades }\end{array}$ \\
\hline 1 & 36 & A & 20 & 15 & 21 & 42 & 8 & 43 \\
\hline A & 28 & B & 21 & 15 & $\mathrm{G}$ & 43 & 14 & 68 \\
\hline B & 32,70 & $2,5,4,9,12,13$ & 22 & 10 & 29 & 44 & 13 & 1 \\
\hline 2 & 14 & 3 & 23 & 18 & $\mathrm{G}$ & 45 & 3 & 46 \\
\hline 3 & 14 & C & 24 & 18 & 37 & 46 & 20,50 & 47, H \\
\hline 4 & 9 & $\mathrm{C}$ & 25 & 15 & $26, \mathrm{E}$ & 47 & 8,50 & $\mathrm{H}$ \\
\hline 5 & 17 & 6 & 26 & 20 & $\mathrm{G}$ & 48 & 35 & 46 \\
\hline 6 & 18 & 7 & E & 30 & $\mathrm{G}$ & 49 & 14 & $50, \mathrm{H}$ \\
\hline 7 & 13 & 8 & 28 & 15 & 27 & 50 & 13 & 51 \\
\hline 8 & 13 & 10 & 27 & 15 & $\mathrm{G}$ & 51 & 9 & 1 \\
\hline 9 & 11 & $\mathrm{C}$ & 29 & 9 & 40 & 52 & 12 & 53 \\
\hline 10 & 12 & 11 & 30 & 40 & G & 53 & 10 & 54 \\
\hline 11 & 9 & 14 & 31 & 39 & $\mathrm{G}$ & 54 & 11 & $\mathrm{H}$ \\
\hline 12 & 7 & 14 & 32 & 14 & 33 & $\mathrm{H}$ & 28,10 & I \\
\hline 13 & 11 & C & 33 & 14 & $\mathrm{G}$ & 55 & 30 & 57 \\
\hline 14 & 21 & 15 & 34 & 4 & $\mathrm{~F}$ & 56 & 4 & 57 \\
\hline 15 & 11 & C & 35 & 11 & 36 & 57 & 34 & 67 \\
\hline C & 34,10 & 16 & 36 & 11 & 40 & 58 & 4 & 60,61 \\
\hline 16 & 35 & 17 & 37 & 12 & $\mathrm{G}$ & 59 & 13 & 61 \\
\hline 17 & 30 & 18 & $\mathrm{~F}$ & 35,90 & 38 & 60 & 13 & I \\
\hline 18 & 17,50 & 19 & 38 & 10 & 39 & 61 & 4 & I \\
\hline 19 & 17,50 & D & 39 & 7 & $\mathrm{G}$ & 62 & 4 & 1 \\
\hline \multirow[t]{10}{*}{$\mathrm{D}$} & 35,80 & - & 40 & 7 & $\mathrm{G}$ & 63 & 14 & I \\
\hline & & & 41 & 7 & $\mathrm{G}$ & 64 & 4 & I \\
\hline & & & $\mathrm{G}$ & 47,45 & - & 65 & 8,25 & 66 \\
\hline & & & & & & 66 & 8,75 & I \\
\hline & & & & & & 67 & 37 & 1 \\
\hline & & & & & & 68 & 47,45 & I \\
\hline & & & & & & 1 & 30 & 69 \\
\hline & & & & & & 69 & 25 & 70 \\
\hline & & & & & & 70 & 12 & 71 \\
\hline & & & & & & 71 & 38 & - \\
\hline
\end{tabular}


apresentamos as tarefas (com seus respectivos tempos) e relações de precedência para cada uma das quatro sublinhas estudadas.
3.2.2 A modelagem específica para o estudo de caso 2

Para o estudo de caso 2, diferentemente do estudo de caso 1 mostrado, só existem tarefas manuais e portanto

Tabela 2: Tempo médio das tarefas relações de precedências das sublinhas 1, 2, 3 e 6.

\begin{tabular}{|c|c|c|c|c|c|c|c|c|c|c|c|}
\hline \multicolumn{3}{|c|}{ SUBLINHA 1} & \multicolumn{3}{|c|}{ SUBLINHA 2} & \multicolumn{3}{|c|}{ SUBLINHA 3} & \multicolumn{3}{|c|}{ SUBLINHA 6} \\
\hline $\begin{array}{l}\text { Código } \\
\text { Tarefa }\end{array}$ & $\begin{array}{c}\text { Tempo } \\
\text { Tarefa } \\
\text { (seg) }\end{array}$ & $\begin{array}{c}\text { Precede } \\
\text { diretamente } \\
\text { atividades }\end{array}$ & $\begin{array}{l}\text { Código } \\
\text { Tarefa }\end{array}$ & $\begin{array}{c}\text { Tempo } \\
\text { Tarefa } \\
\text { (seg) }\end{array}$ & $\begin{array}{c}\text { Precede } \\
\text { diretamente } \\
\text { atividades }\end{array}$ & $\begin{array}{l}\text { Código } \\
\text { Tarefa }\end{array}$ & $\begin{array}{c}\text { Tempo } \\
\text { Tarefa } \\
\text { (seg) }\end{array}$ & $\begin{array}{c}\text { Precede } \\
\text { diretamente } \\
\text { atividades }\end{array}$ & $\begin{array}{l}\text { Código } \\
\text { Tarefa }\end{array}$ & $\begin{array}{c}\text { Tempo } \\
\text { Tarefa } \\
\text { (seg) }\end{array}$ & $\begin{array}{c}\text { Precede } \\
\text { diretamente } \\
\text { atividades }\end{array}$ \\
\hline 1 & 24 & 2,3 & 21 & 5 & 22 & 42 & 24 & 43 & 74 & 9 & 75 \\
\hline 2 & 2 & 5 & 22 & 5 & 23 & 43 & 9 & 44 & 75 & 8 & 76 \\
\hline 3 & 5 & 4 & 23 & 6 & 24 & 44 & 15 & $45,46,47$ & 76 & 7 & 77, 78 \\
\hline 4 & 11 & 5 & 24 & 7 & 25 & 45 & 5 & - & 77 & 7 & 79 \\
\hline 5 & 6 & $6,9,12$ & 25 & 9 & 26 & 46 & 6 & - & 78 & 6 & $\begin{array}{c}79,80,81 \\
82,83\end{array}$ \\
\hline 6 & 6 & 6 & 26 & 7 & 27 & 47 & 13 & - & 79 & 11 & $89,90,91$ \\
\hline 7 & 6 & 8,14 & 27 & 15 & 28,30 & 48 & 9 & 51 & 80 & 7 & $89,90,91$ \\
\hline 8 & 8 & 19 & 28 & 6 & 29 & 49 & 11 & - & 81 & 7 & $89,90,91$ \\
\hline 9 & 6 & 10 & 29 & 13 & 37 & 50 & 5 & 51 & 82 & 7 & $89,90,91$ \\
\hline 10 & 6 & 11,18 & 30 & 7 & 31 & 51 & 3 & 53 & 83 & 7 & $89,90,91$ \\
\hline 11 & 8 & 19 & 31 & 8 & 32 & 52 & 9 & - & 84 & 2 & 95 \\
\hline 12 & 6 & $13,15,16,17$ & 32 & 3 & 33 & 53 & 3 & - & 85 & 5 & 95 \\
\hline 13 & 10 & 20 & 33 & 6 & 34,35 & 54 & 24 & 55 & 86 & 7 & 95 \\
\hline 14 & 6 & 19 & 34 & 8 & 36 & 55 & 4 & 57 & 87 & 2 & 95 \\
\hline 15 & 9 & 19 & 35 & 4 & 37 & 56 & 7 & - & 88 & 5 & 95 \\
\hline 16 & 4 & 19 & 36 & 9 & 37 & 57 & 13 & 73 & 89 & 5 & - \\
\hline 17 & 9 & 19 & 37 & 4 & 38,39 & 58 & 6 & 60 & 90 & 6 & - \\
\hline 18 & 6 & 19 & 38 & 12 & 40 & 59 & 5 & - & 91 & 6 & - \\
\hline 19 & 5 & - & 39 & 16 & 40 & 60 & 10 & - & 92 & 8 & 95 \\
\hline \multirow[t]{13}{*}{20} & 12 & 19 & 40 & 6 & 41 & 61 & 4 & 63 & 93 & 8 & 95 \\
\hline & & & 41 & 13 & - & 62 & 2 & - & 94 & 2 & 96 \\
\hline & & & & & & 63 & 7 & - & 95 & 9 & $\begin{array}{c}97,98,99 \\
101\end{array}$ \\
\hline & & & & & & 64 & 7 & - & 96 & 6 & 95 \\
\hline & & & & & & 65 & 7 & 66,68 & 97 & 5 & 102,103 \\
\hline & & & & & & 66 & 6 & - & 98 & 2 & 104 \\
\hline & & & & & & 67 & 2 & - & 99 & 5 & 100 \\
\hline & & & & & & 68 & 4 & 69 & 100 & 4 & 102 \\
\hline & & & & & & 69 & 6 & 70 & 101 & 6 & 102 \\
\hline & & & & & & 70 & 5 & 71 & 102 & 7 & 104 \\
\hline & & & & & & 71 & 2 & - & 103 & 8 & 104 \\
\hline & & & & & & 72 & 10 & - & 104 & 16 & - \\
\hline & & & & & & 73 & 4 & - & & & \\
\hline
\end{tabular}


o conjunto de restrições modeladas na seção 3.1 .3 não é necessário. Portanto para o caso 2 o modelo utilizado foi exatamente o modelo proposto por Fernandes \& Morábito (1993).

\section{RESULTADOS E AVALIAÇÃO}

Nesta seção são mostrados os resultados da aplicação do método exato proposto. Para ambos os estudos de caso o modelo matemático foi programado no software de programação matemática GAMS-CPLEX 19.3. Os modelos foram executados em um processador Pentium IV.

\section{A linha de montagem de eletrodomésticos da Alinha branca (estudo de caso 2) é dividida em}

\section{6 sublinhas num total de 41 estações de trabalho.}

Como estamos tratando de um SALBP-1, para se avaliar o grau de melhoria que o balanceamento por meio deste método oferece em relação ao balanceamento utilizado atualmente pelas empresas, vamos verificar o impacto nas duas funções objetivos que são equivalentes nos SALBP-1:

a) O número de estações de trabalho

b) O tempo ocioso total ( o qual pode também ser avaliado pelo desbalanceamento de linha)

O tempo ocioso de uma estação de trabalho é a diferença entre o tempo de ciclo e o tempo da estação de trabalho. O tempo ocioso total da linha é a somatória dos tempos ociosos das estações de trabalho que compõem a linha. Matematicamente temos:

Tempo ocioso total da linha $=K C-\sum_{i=1}^{N} p_{i}$

onde :

$K=$ número de estações de trabalho

$C=$ tempo de ciclo (não pode ser menor que o tempo de montagem do posto mais lento)

$\sum_{i=1}^{N} p_{i}=$ tempo total de trabalho requerido por um item

Uma medida semelhante ao tempo ocioso total da linha e que utilizaremos nos resultados mostrados é o chamado desbalanceamento de linha. O desbalanceamento de linha (D) é a relação entre o tempo ocioso total da linha e o tempo total gasto pelo produto para se mover do início ao final da linha (KC). Matematicamente temos:

$$
\text { Desbalanceamento de linha }(D)=\frac{K C-\sum_{i=1}^{N} p_{i}}{K C}
$$

Por meio do desbalanceamento de linha podemos avaliar a eficiência da linha, uma vez que ambas as medidas são complementares:

Eficiência da linha $=(1-$ Desbalanceamento de linha $)$

\subsection{Resultados referentes ao estudo de caso 1}

Para o estudo de caso 1 o resultado do balanceamento de linha gerado por meio da utilização do método exato proposto é o seguinte. Tarefas alocadas à estação de trabalho 1: tarefa 1 ; à estação 2: tarefa $\mathrm{A}$; à estação 3 : tarefa B; à estação 4: tarefas 5,6 e 9; à estação 5: tarefas 2, 7, 8 e 12; à estação 6: tarefas 3, 4, 10 e 13; à estação 7: tarefas 11, 14 e 15; à estação 8: tarefa C; à estação 9: tarefa 16; à estação 10: tarefa 17; à estação 11: tarefas 18 e 19: à estação 12: tarefa $\mathrm{D}$. Também avaliamos para cada sublinha se houve ou não melhorias com relação ao balanceamento atualmente existente em relação ao número de estações de trabalho e ao desbalanceamento da linha.

Atualmente a sublinha 1 conta com 13 estações de trabalho, das quais 9 são manuais e 4 são automáticas. A solução gerada pelo modelo é de 12 estações de trabalho. Portanto, vemos que houve possibilidade de redução de 1 posto de trabalho e portanto também uma redução no desbalanceamento da linha:

$$
\begin{gathered}
\mathrm{D}_{\text {atual }}=(13 \times 47,45-446,6) / 616,85=27,6 \% \\
\mathrm{D}_{\text {proposto }}=(12 \times 47,45-446,6) / 569,40=21,5 \%
\end{gathered}
$$

No caso da sublinha 2 tivemos $=>$ Tarefas alocadas à estação de trabalho 1: tarefas 22, 23, 32 e 34; à estação 2: tarefa F; à estação 3: tarefas 30 e 41; à estação 4: tarefas 20, 21, 38 e 39; à estação 5: tarefas 25, 26 e 35; à estação 6: tarefas 24,28 e 33; à estação 7: tarefas 27, 29, 36 e 37; à estação 8: tarefas 31 e 40; à estação 9: tarefa E; à estação 10: tarefa G.

Atualmente a sublinha 2 conta com 12 estações de trabalho, das quais 9 são manuais e 3 são automáticas. A solução gerada pelo modelo é de 10 estações de trabalho. Portanto, vemos que houve uma redução de 2 postos de trabalho e portanto também uma redução no desbalanceamento da linha:

$$
\begin{gathered}
\mathrm{D}_{\text {atual }}=(12 \times 47,45-439,35) / 569,4=22,8 \% \\
\mathrm{D}_{\text {proposto }}=(10 \times 47,45-439,35) / 474,5=7,4 \%
\end{gathered}
$$


No caso da sublinha 3 tivemos $=>$ Tarefas alocadas à estação de trabalho 1: tarefas 52, 53, 54 e 64; à estação 2: tarefas 44, 49, 58 e 60; à estação 3: tarefas 42,45 e 48; à estação 4 : tarefas 43,46 e 47; à estação 5 : tarefa 68; à estação 6: tarefas $59,61,62,63,66$; à estação 7 : tarefa $\mathrm{H}$; à estação 8: tarefas 50 , 55 e 56; à estação 9: tarefa 57; à estação 10: tarefas 51 e 67; à estação 11: tarefa I; à estação 12: tarefas 69 e 70; à estação 13: tarefa 71

Atualmente, a sublinha 3 conta com 15 estações de trabalho, das quais 13 são manuais e 2 são automáticas. A solução gerada pelo modelo é de 13 estações de trabalho. Portanto vemos que houve uma redução de 2 postos de trabalho e portanto também uma redução no desbalanceamento da linha:

$$
\begin{gathered}
\mathrm{D}_{\text {atual }}=(15 \times 47,45-529,55) / 711,75=25,6 \% \\
\mathrm{D}_{\text {proposto }}=(13 \times 47,45-529,55) / 616,85=14,1 \%
\end{gathered}
$$

\subsection{Resultados referentes ao estudo de caso 2}

Para o estudo de caso 2, sublinha 1, o resultado do balanceamento de linha gerado por meio da utilização do método exato proposto é o seguinte. Tarefas alocadas à estação de trabalho 1: tarefa 1; à estação 2: tarefas $2,3,4$ e 5; à estação 3: tarefas 6, 7, 9 e 14; à estação 4: tarefas 8,12 e 17; à estação 5: tarefas 10 e 13; à estação 6: tarefas 11 e 20; à estação 7 : tarefas 15, 16, 18 e 19.

Para a sublinha 1 há a possibilidade de redução de 1 posto de trabalho e portanto uma redução no desbalanceamento da linha:

$$
\begin{gathered}
\mathrm{D}_{\text {atual }}=(8 \times 24-155) / 192=19,3 \% \\
\mathrm{D}_{\text {proposto }}=(7 \times 24-155) / 168=7,7 \%
\end{gathered}
$$

Para a sublinha 2 o resultado do balanceamento de linha é o seguinte. Tarefas alocadas à estação de trabalho 1: tarefas 21,22 e 23; à estação 2 : tarefas 24,25 e 26 ; à estação 3 : tarefa 27; à estação 4: tarefas 28 e 29; à estação 5: tarefas 30, 31, 32 e 33; à estação 6: tarefas 34 e 36; à estação 7: tarefas 35, 37 e 38; à estação 8: tarefas 39 e 40; à estação 9: tarefa 41.

Para a sublinha 2 não há possibilidade de redução de posto de trabalho e portanto o desbalanceamento permanece o mesmo, ou seja:

$$
\mathrm{D}_{\text {atual }}=\mathrm{D}_{\text {proposto }}=(9 \times 24-169) / 216=21,76 \%
$$

Com relação à sublinha 3 temos o seguinte: tarefas alocadas à estação de trabalho 1: tarefa 42; à estação 2 : tarefas 43 e 44; à estação 3: tarefas 45,46 e 47; à estação 4 : tarefas 50, 58, 69, 70 e 71; à estação 5: tarefas 48, 64 e 65; à estação 6: tarefa 54; à estação 7: tarefas 51, 53, 60, 66 e 67; à estação 8: tarefas 52, 55, 56 e 68; à estação 9: tarefas 57 e 72 ; à estação 10: tarefas 59, 61, 62, 63 e 73; à estação 11: tarefa 49.

Para a sublinha 3 há a possibilidade de redução de 1 posto de trabalho e portanto uma redução no desbalanceamento:

$$
\begin{aligned}
& D_{\text {antes }}=(12 \times 24-247) / 12 \times 24=14,2 \% \\
& D_{\text {depois }}=(11 \times 24-247) / 11 \times 24=6,4 \%
\end{aligned}
$$

Com relação à sublinha 6 temos o seguinte: tarefas alocadas à estação de trabalho 1: tarefas 74,75 e 76 ; à estação 2 : tarefas 77, 78 e 79; à estação 3 : tarefas 80,81 e 83 ; à estação 4: tarefas 82, 84, 85 e 86; à estação 5: tarefas 87, 90, 92, 93 e 94; à estação 6: tarefas 88,89 e 91; à estação 7: tarefas 95, 96, 99 e 100; à estação 8: tarefas 97, 98, 101 e 102; à estação 9: tarefas 103 e 104.

Para a sublinha 6 há a possibilidade de redução de 3 postos de trabalho e, portanto, uma redução no desbalanceamento, como mostrado a seguir:

$$
\begin{gathered}
\mathrm{D}_{\text {antes }}=(12 \times 24-200) / 12 \times 24=30,6 \% \\
\mathrm{D}_{\text {depois }}=(9 \times 24-200) / 9 \times 24=7,4 \%
\end{gathered}
$$

Tabela 3: resumo de melhorias conseguidas nos dois estudos de caso realizados.

\begin{tabular}{cccccc}
\hline & \multicolumn{2}{c}{ ESTUDO DE CASO 1 } & \multicolumn{2}{c}{ ESTUDO DE CASO 2 } \\
\hline Sublinha & $\begin{array}{c}\text { Número atual de } \\
\text { estações de trabalho }\end{array}$ & $\begin{array}{c}\text { Número proposto de } \\
\text { estações de trabalho }\end{array}$ & Sublinha & $\begin{array}{c}\text { Número atual de } \\
\text { estações de trabalho }\end{array}$ & $\begin{array}{c}\text { Número proposto de } \\
\text { estações de trabalho }\end{array}$ \\
\hline 1 & 13 & 12 & 1 & 8 & 7 \\
2 & 12 & 10 & 2 & 9 & 11 \\
3 & 15 & 13 & 3 & 12 & 9 \\
\hline Total Caso 1 & 40 & & 6 & 12 & 36 \\
\hline
\end{tabular}




\section{CONCLUSÕES}

O presente trabalho trata do problema de balanceamento de linha de montagem simples que tem como critério de otimização a minimização do tempo ocioso total para um dado tempo de ciclo fixo, o que é equivalente a minimizar o número de estações de trabalho (SALBP-1).

$\mathrm{O}$ trabalho contribui à medida que realiza uma aplicação de modelos de otimização para balanceamento de linha de montagem em duas situações práticas (GALBP com 80 tarefas e SALBP com 104 tarefas), mostrando resultados auspiciosos com relação à melhoria da eficiência das linhas pesquisadas. A tabela 3 resume as melhorias nas linhas de montagem nos dois estudos de caso realizados (redução de 40 para 35 estações e de 41 para 36 estações). Esta redução foi possível no caso 1 nas estações manuais; cada estação automática realiza apenas uma tarefa que não pode ser eliminada nem realocada a uma outra estação. A redução no número total de estações utilizadas se deve ao fato de que o modelo matemático analisa (de forma implícita) um número de alternativas muitíssimo maior que o processista de cada uma das duas empresas.

Como contribuição deste artigo, também vale a pena destacar que foi modelada uma nova restrição (caso 1), inédita na literatura (um survey bastante recente sobre o GALBP, encontrado em Becker \& Scholl (2006) não mostra esta situação específica), que leva em conta linhas de montagem que tenham operações manuais e automáticas. Esta situação pode ser utilizada na prática em problemas de balanceamento de linha que apresentam esta situação.

Outra conclusão importante é a seguinte: problemas de balanceamento de linhas de montagem, reconhecidamente pertencentes à classe NP-árdua, possuem instâncias de interesse prático, por exemplo os dois casos aqui tratados, que podem ser resolvidas de forma exata, isto é, sem utilização de procedimentos heurísticos.

Como pesquisas futuras podem ser indicadas, por exemplo: (i) estudar o efeito da mudança na taxa de produção (e portanto no tempo de ciclo) sobre o rebalanceamento da linha; (ii) analisar se o rebalanceamento é mais fácil de ser tratado por modelagem de otimização do que o problema inicial de balanceamento; (iii) confirmar na prática (por meio de um survey) que situações reais possuem cerca de 100 tarefas e que estão tecnicamente divididas em alguns ( 3 a 6 ) grupos de sublinhas (chamadas de células na nomenclatura das empresas). Se isso ocorrer, a grande aplicabilidade do método estará comprovada; se isso não ocorrer, poder-se-á fazer uma previsão do maior sucesso da proposta em face dos desenvolvimentos que vêm acontecendo com os solvers do software GAMS e com o desenvolvimento do hardware dos micro-computadores existentes nas empresas para dar suporte ao problema de balanceamento de linha.

\section{Artigo recebido em 18/04/2006 Aprovado para publicação em 04/03/2008}

\section{REFERÊNCIAS}

AMEN, M. Heuristic methods for cost-oriented assembly line balancing: A comparison on solution quality and computing time. International Journal Production Economics, v. 69, p. 255-264, 2001.

Heuristic methods for costoriented assembly line balancing: A survey. International Journal Production Economics, v. 68 , p. $1-14,2000$

Cost-oriented assembly line balancing - model formulations, solution difficulty, upper and lower bounds. European Journal of Operational Research, v. 168, p. 747-770, 2006.
BAYBARS, I. A survey of exact Algorithms for the Simple Assembly Line Balancing Problem; Management Science, v. 32, n. 8, 1986.

BECKER, C.; SCHOLL, A. A survey on problems and methods in generalized assembly line balancing. European Journal of Operational Research, v. 168, n. 3, p. 694-715, 2006.

BOWMAN, E. H. Assembly line balancing by linear programming, Operations Research, v. 8, p. 385-389, 1960.

DAR-EL, E. M. MALB-A heuristic technique for balancing large single-model assembly lines, AlIE Transactions, v. 5, p. 343-356, 1973.
EREL, E.; SARIN, S. C. A survey of the Simple Assembly Line Procedures; Production Planning \& Control, v. 9, n. 5, p. 414-434, 1998.

FERNANDES, F. C. F.; MORÁBITO, R. Linguagens de Modelagem GAMS e LINGO: Aplicação a um problema de Balanceamento de Linha de Montagem; Cadernos de Engenharia de Produção, Ano X, n. 20, p. 8-31, 1993.

GHOSH, S.; GAGNON, R. J. A comprehensive literature review and analyses of design, balancing and scheduling of assembly systems; International Journal of Production Research, v. 27, n. 4, p. 637-670, 1989. 
HELGESON, W.B.; SALVESON, M.E.; SMITH, W.W. How to balance an assembly line. Management Report n. 7 (New Caraan, Conn.: Carr Press, Division for Advanced Management), 1954

MASTOR, A. A. An experimental investigation and comparative evaluation of production line balancing techniques, Management Science, v. 16, p. 728-746, 1970.

PATTERSON, J. H.; ALBRACHT, J. J. Assembly line balancing: 0-1 programming with Fibonacci Search, Operations Research, v. 23, p. 166-174, 1975.
PLANS, J.; COROMINAS, A. Modelling and solving the SALB-E problem. Proceedings of the IEEE International Symposium on Assembly and Task Planning. Porto, Portugal, p. 356-360, 1999.

SALVESON, M. E. The assembly line balancing problem, Journal Industrial Engineering, v. 6, p. 18-25, 1955.

SCHOLL, A. Balancing and sequencing assembly lines, $2^{\text {nd }}$ ed. Physica, Heidelberg, 1999.

SCHOLL, A.; BECKER, C. State-of-the-art exact and heuristic solution procedures for simple assembly line balancing. European Journal of
Operational Research, v. 168, n. 3, p. 666-693, 2006.

TALBOT, F. B.; PATTERSON, J. H.; GEHRLEIN W.V. A comparative evaluation of heuristic line balancing techniques, Management Science, v. 32, n. 4, p. 430-454, 1986.

THANGAVELU, S. R.; SHETTY, C. M. Assembly Line Balancing by Zero-One Programming. AlIE Transactions, v. 3, n. 1, p. 61-68, 1971.

WHITE, W. W. Comments on a paper by Bowman, Operations Research, v. 9, p. 274-276, 1961.

\section{AGRADECIMENTOS}

Os autores agradecem as sugestões dos dois referees anônimos para a melhoria do artigo.

\section{SOBRE OS AUTORES}

\section{Flávio César Faria Fernandes}

Departamento de Engenharia de Produção

Universidade Federal de São Carlos

End.: Rod. Washington Luís, Km 235 - CEP 13565-905 - São Carlos - SP

Tel.: (16) 3351-8237 ramal 9214

E-mail: dfcf@power.ufscar.br

\section{Moacir Godinho Filho}

Departamento de Engenharia de Produção

Universidade Federal de São Carlos

Tel.: (16) 3351-8237 ramal 9235

E-mail:moacir@dep.ufscar.br

\section{Ricardo Augusto Cutigi}

Universidade Federal de São Carlos

\section{Aline Malerbo Guiguet}

Universidade Federal de São Carlos 